\title{
Comparing Anterior Cervical Discectomy Fusion with Cervical Disc Arthroplasty in Cervical Spondylosis
}

\section{Siraj Gibani, MD1; Jeffrey Badday, $\mathrm{MD}^{2}$}

${ }^{1}$ Inland Neurosurgery Institute, Pomona, CA

${ }^{2}$ Casa Colina Hospital, Department of Physical Medicine and Rehabilitation, Pomona, CA

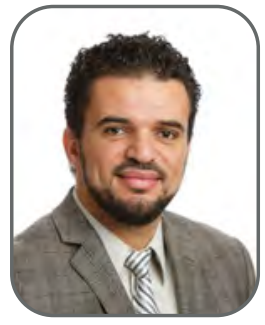

Siraj Gibani, MD

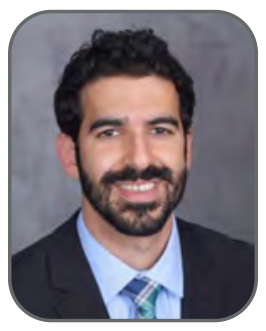

Jeffrey Badday, MD

\section{KEYWORDS:}

Adjacent Level Disease, Anterior Cervical Discectomy and Fusion, Cervical Spondylosis, Cervical Disc Arthroplasty, Clinical Outcomes, Complications, Heterotropic Ossification, Implant Migration, Subsidence.

\section{ABSTRACT}

The present article reviews updated research, patient selection, complications associated with types of cervical disc implants, and its use in patients with multilevel cervical spondylosis. Recent metanalysis comparing Cervical Disc Arthroplasty (CDA) and Anterior Cervical Discectomy and Fusion (ACDF) has demonstrated safety and equal or superior clinical outcomes based on SF-36 NDI and VAS scores. Recent studies show similar outcomes for using CDA in two-level cervical disc disease of those suffering radiculopathy and/or /myelopathy. Long-term studies are more available to show decreased rates of adjacent segment disease and reoperation rates following cervical disc replacement. Cervical arthroplasty continues to be a promising technique for which now there is growing level 1a evidence. Further long-term data is awaited to investigate two-level CDA and reoperation rates specifically due to adjacent level disease, subsidence, implant migration, and heterotropic ossification.

\section{INTRODUCTION}

Neck pain is a common clinical complaint with an estimated 10-20\% prevalence in the adult population. Cervical degenerative changes known as arthritis of the cervical spine (cervical spondylosis) is a common cause of chronic neck pain, often accumulating as wear and tear of the discs, facet joints, and uncovertebral joints, and vertebral bodies over time that eventually leads to cervical instability. Cervical spondylosis is the most common cause of acute and chronic neck pain in adults aged 40-years-or-older and can begin as early as the first decade of life. ${ }^{1}$ Further review of the global burden of low back and neck pain estimated that in 2015, more than a third of a billion people worldwide had mechanical neck pain of at least three months' duration. ${ }^{2}$ The estimated annual incidence for those with cervical spondylosis who developed cervical radiculopathy is approximately 83 cases per 100,000 persons. This rate is similar to a previous incidence rate found in a 14-year general population study held in Rochester, MN, with C7 being the most common cervical spinal nerve root site of impingement., ${ }^{3,4}$ Typical standard treatment for cervical spondylosis has been conservative therapy with or without steroid injections; however, when it leads to cervical instability and 
intractable neck pain, other interventions explored are Anterior Cervical Discectomy and Fusion (ACDF), Posterior Cervical Fusion (PCF), Cervical Circumferential Fusion (CCF), and Cervical Disc Arthroplasty (CDA). From the New York Statewide Planning and Research Cooperative System Database, one study analyzed 87,045 primary cervical fusions from 1997 to 2012 and found the annual cervical fusion rate increased from 23.7 to 50.6 per 100,000 population. Of the most common procedures performed, ACDF made up $85 \%$ of the surgeries, followed by PCF (12.3\%) and then CCF (2.5\%). The mean age was 50-years-old plus or minus ten years for the ACDF group with an in-hospital complication rate of $3 \%$ (lower than PCF and CCF). ${ }^{5}$ Although ACDF continues to be the standard intervention for advanced cervical degenerative disc disease, CDA has become the preferable option in selected patients with one to twolevel symptomatic cervical degenerative disc disease. ${ }^{6}$ Over the past decade, several RCT trials in a 2016 metaanalysis showed growing level one evidence for cervical disc arthroplasty to be equally as safe and effective as ACDF in patients suffering cervical myelopathy and radiculopathy with cervical spondylosis as the main culprit.? Now, with new technological advances in CDA that decrease operating time with easier surgical placement, there are less secondary reoperations with CDA than ACDF. ${ }^{8}$ With the proper patient selection, this allows patients undergoing CDA to maintain cervical range of motion without compromising cervical stability. In addition to preserving cervical range of motion, longterm studies are beginning to show lower incidence of adjacent level disease, lower reoperation rates, and overall increased patient satisfaction in favor of CDA than ACDF. 910,14

Even though CDA is becoming more favorable, postoperative complications do arise with certain devices, and the decision to reoperate depends on symptom progression and cervical instability captured on radiographic imaging. Common complications encountered after CDA and ACDF procedures include heterotropic ossification, adjacent level disease, subsidence, kyphosis, pseudoarthrosis, implant migration, dysphagia, and transient dysphonia. To avoid and minimize the risk of these complications, it is essential to review patient selection criteria and the current FDAapproved cervical devices on the market.

\section{FIGURE $1 .{ }^{19}$}

MRI of patients with Degenerative Cervical Myelopathy. T2WI (A) and T1WI (B) studies are showing a Schmorl's node at the superior endplate of C-7 (S), OPLL extending from C-4 to C-7 (arrowheads), and hypertrophy of the PLL (H). C: T2WI study showing widespread spondylotic changes of the cervical spine with decreased vertebral height noted in C-4 (double arrow) and a large posterior osteophyte (0).

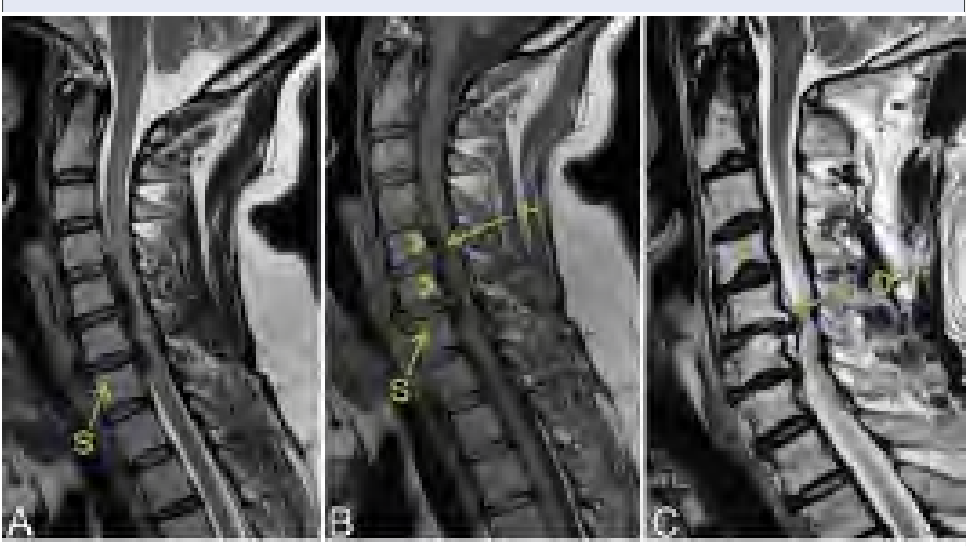

\section{FIGURE 2.}

Lateral cervical X-ray illustrating cervical spondylosis with disc space narrowing at $\mathrm{C} 5 / \mathrm{C} 6$ and $\mathrm{Cb} / \mathrm{C} 7$ with endplate sclerosis and osteophyte complex at the uncovertebral joints. (Orthobullets.com)

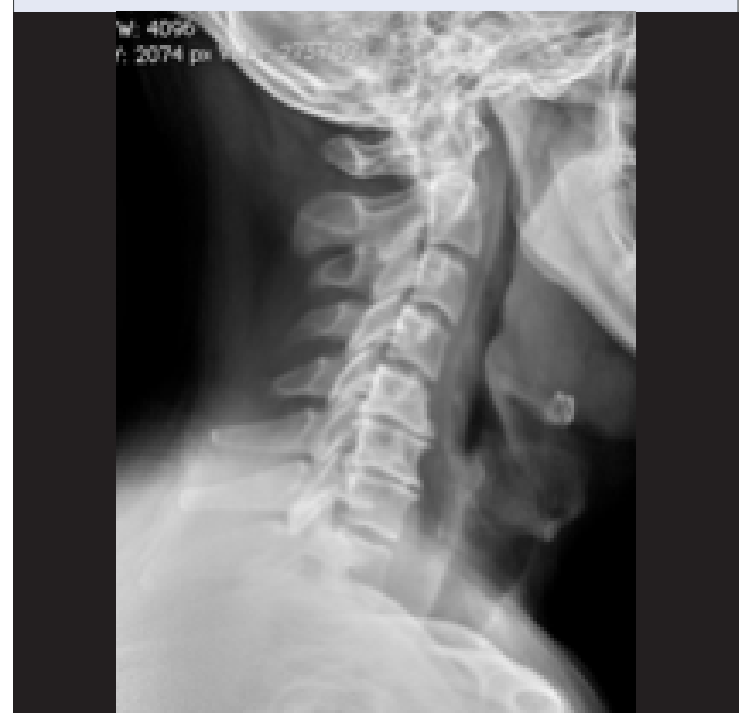




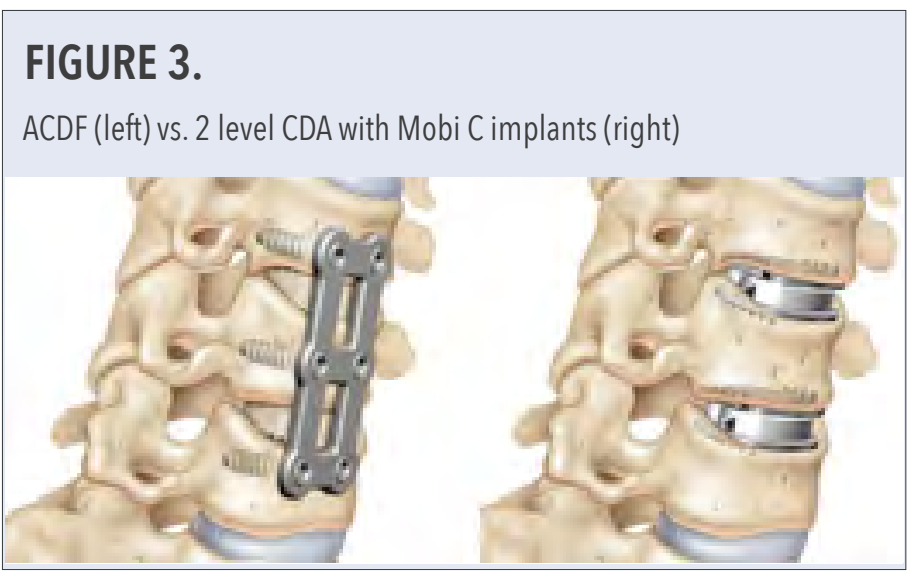

\section{INDICATION AND PATIENT SELECTION}

Chronic debilitating neck pain can be subjectively characterized with the NDI and SF-36 outcome measures. The NDI incorporates impaired function and sleep in which the SF-36 does not have in the overall pain assessment. Several studies compare ACDF with CDA in which patients in both groups have average pre-surgery scores with an NDI of 30 and SF-36 of 40 . These baseline scores can be utilized as a screen before clinically evaluating cervical radiculopathy and/or myelopathy. If deemed surgical candidates, CDA can be a viable option for ongoing cervical radiculopathy and/ or myelopathy. Surgical planning must include radiographic evidence in the form of cervical $x$-rays and MRIs to show the severity of spinal nerve root impingement and/or spinal cord compression (Figures $1 \& 2$ ).

In some cases, which may be difficult to localize the pain generator, an EMG/NCS can help rule in cervical radiculopathy at the most affected level. Targeted index levels for implant placement should be anywhere between C3-C7 with minimal radiographic evidence of adjacent level disease. Quicker recovery is seen in patients without a history of smoking, alcohol abuse, mental illness, chronic steroid use, diabetes mellitus, BMI of greater than 40 , autoimmune diseases (RA, Lupus, Scleroderma), loss of cervical lordosis (cervical kyphosis or scoliosis), previous neck and back surgeries, lumbar spondylosis, invasive cancer, and metal allergies. Fewer complications with pseudoarthrosis, implant migration, subsidence, adjacent level disease, and kyphosis arise if the patient has no history of metabolic bone disease and osteoporosis. These factors can be screened with a DEXA scan and X-ray to assess bone quality and early signs of cervical degeneration at the adjacent levels. For more advanced cervical spondylosis, patients should not have disc heights less than $3 \mathrm{~mm}$ at the adjacent levels and have a Kellgren Lawrence

\section{FIGURE 4.}

Prestige ST (a), Bryan (b), Prodisc C (c)

a

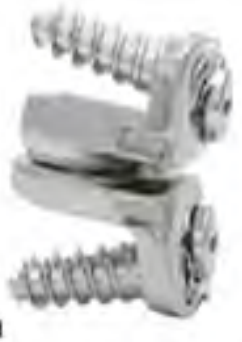

b
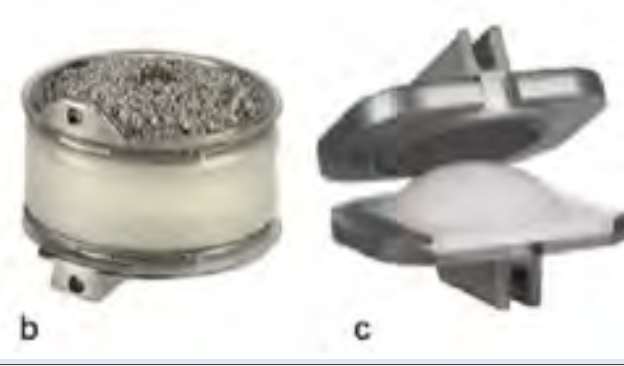

Classification with a grade II or more to reduce postoperative complications as mentioned above. With strict and consistent inclusion and exclusion criteria in future studies, it will be easier for the surgeon to analyze implant advantages and disadvantages tailored to each individual.

\section{CDA DEVICES AND HOW THEY WORK}

Most implants have either single or double articulation surfaces, with the first generation of implants having a geometry of articulations including ball-and-socket, balland-trough, biarticulating, and saddle designs. Apart from the saddle designs, almost all other devices employ a spherical interface with or without translation. Independent translation (distinct from rotatory translation) allows for a mobile center of rotation (COR).$^{18}$ Artificial cervical disc replacements that allow for a mobile center of rotation have a theoretical advantage in providing normal kinematics over a range of device positions. ${ }^{15}$ Cervical disc replacements with a ball and socket design (Pro-Disc C) provide a fixed center of rotation and require precise device placement to restore normal kinematics.

Thus far, single cervical disc level long-term data have been published on the Prestige ST, Bryan disc, and Pro-Disc $C$ disc for single-level cervical arthroplasty showing equivalent efficacy to ACDF. ${ }^{11,12,13}$ (Figure 4). A recent five-year study investigating Mobi- $\mathrm{C}$ for twolevel cervical arthroplasty showed that multilevel is just as safe as single-level CDA. ${ }^{6}$ (Figure 3 ) Mobi-C is a low-profile, mobile-bearing CDA device composed of two cobalt-chromium alloy endplates and an ultra-high molecular weight polyethylene mobile core in a metalon-polyethylene bearing surface (Figure 5). In theory, by preserving the motion of the operated segment, CDAs place comparatively less stress on adjacent levels, which may serve to protect those levels. ${ }^{16}$ Each CDA 
design is different with complications associated with patient selection. The CDA devices have variability with keels, teeth, spikes, rails, and screws, which are utilized to achieve immediate stability of the implant. Larger keels \& teeth require cuts into cortical and cancellous bone, leading to a risk of releasing osteoinductive factors promoting subsidence, heterotropic ossification, and fusion across the disc space. ${ }^{17}$ Subsidence, which pertains to the sinking of the endplates into bone, is one reason to avoid CDA in osteopenic/osteoporotic patients based on large keel designs and excessive bone chiseling before implant fixation between the vertebral bodies. Some of the designs do not have invasive keels or screws like the Mobi-C (Figure 6). No bone chiseling is required for Mobi-C placement, which reduces operative steps and preserves the vertebrae for a stable surface ideal for one or two-level implantation.

\section{CONCLUSION}

Device-specific selection in CDA is often one of the surgeon preferences based on experience and comfort level. The general trend has been towards a wider application of CDA within the patient population. If more studies were consistent with common selection criteria, it would be more effective to compare the different FDA-approved CDA devices on the market. Current studies show a trend that CDA is superior to ACDF; however, more literature is required for long-term follow-up to investigate two-level CDA and reoperation rates specifically due to subsidence, implant migration, heterotropic ossification, and adjacent level disease. Future direction will also influence the market for artificial lumbar disc replacement, which has not shown as favorable data compared to CDA.

\section{AUTHOR DISCLOSURES:}

No relevant financial affiliations or conflicts of interest.

\section{REFERENCES:}

1. Theodore. Degenerative Cervical Spondylosis. N Engl J Med. 2020;383(2):159.

2. Hurwitz EL, Randhawa $K, Y u$ H, Côté $P$, Haldeman S. The Global Spine Care Initiative: a summary of the global burden of low back and neck pain studies. Eur Spine J 2018;27: Suppl 6:796-801].

3. Yoss, Corbin, Maccarty, Love.Significance of symptoms and signs in localization of involved root in cervical disk protrusion. Neurology. 1957;7(10):673.

\section{FIGURE 5.}

Lateral cervical XR demonstrating Mobi $\mathrm{C}$ device.

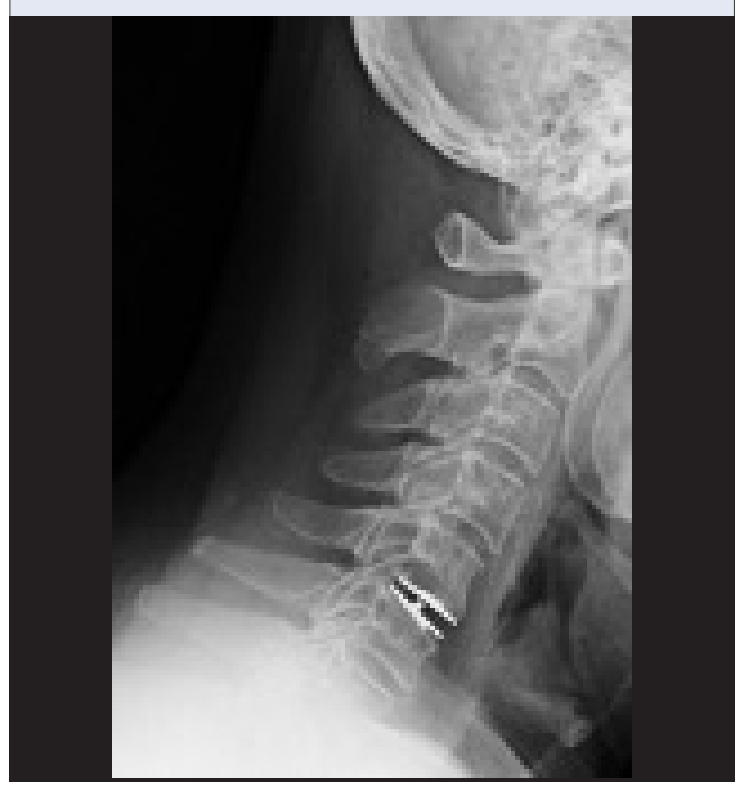

\section{FIGURE 6.}

Mobi $\mathrm{C}$ device with the corresponding axis of rotation $(\mathrm{x} 3)$ and axis of translation ( $\mathrm{x} 2$ ).

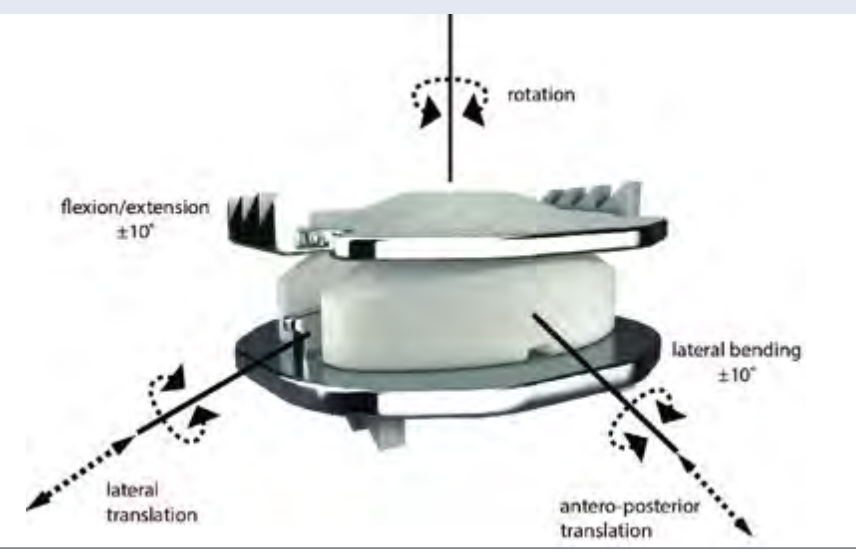

4. Radhakrishnan K, Litchy WJ, O'Fallon WM, Kurland LT. Brain Epidemiology of cervical radiculopathy. A population-based study from Rochester, Minnesota, 1976 through 1990. 1994;117 (Pt 2):325.

5. Stephan N. Salzmann. Peter B. Derman. Cervical Spinal Fusion: 16-Year Trends in Epidemiology, Indications, and In-hospital Outcomes by Surgical Approach. World Neurosurgery. 2018. 
6. Radcliff, Domagoj. Five-year clinical results of cervical total disc replacement compared with anterior discectomy and fusion for treatment of 2-level symptomatic degenerative disc disease: a prospective, randomized, controlled, multicenter investigational device exemption clinical trial. Albert. JNS Spine. 2016.

7. Zhu, Zhang. Cervical disc arthroplasty versus anterior cervical discectomy and fusion for symptomatic adjacent segment disease incidence: a meta-analysis of prospective randomized controlled trials. Spine. 2016.

8. Hilibrand AS, Robbins M. Adjacent segment degeneration and adjacent segment disease: the consequences of spinal fusion? Spine J 2004;4:190s-4s.

9. Hilibrand AS, Carlson GD, Palumbo MA, et al. Radiculopathy and myelopathy at segments adjacent to a previous anterior cervical arthrodesis site. J Bone Joint Surg Am 1999;81:519-28.

10. Delamarter RB, Zigler J: Five-year reoperation rates, cervical total disc replacement versus fusion, results of a prospective randomized clinical trial. Spine (Phila Pa 1976) 38:711-717, 2013.

11. Sasso RC, Metcalf NH, Hipp JA, Wharton ND, Anderson PA: Sagittal alignment after Bryan cervical arthroplasty. Spine (Phila Pa 1976) 36:991-996, 2011.

12. Zigler JE, Delamarter R, Murrey D, Spivak J, Janssen M: ProDisc-C and anterior cervical discectomy and fusion as surgical treatment for single-level cervical symptomatic degenerative disc disease: five-year results of a Food and Drug Administration study. Spine (Phila Pa 1976) 38:203-209, 2013.
13. Burkus JK, Traynelis VC, Haid RW Jr, Mummaneni PV: Clinical and radiographic analysis of an artificial cervical disc: 7-year follow-up from the Prestige prospective randomized controlled clinical trial: Clinical article. J Neurosurg Spine 21:516-528, 2014.

14. Auerbach JD, Anakwenze OA, Milby AH, Lonner BS, Balderston RA: Segmental contribution toward total cervical range of motion: a comparison of cervical disc arthroplasty and fusion. Spine (Phila Pa 1976) 36:E1593-E1599, 2011.

15. Pickett GE, Rouleau JP, Duggal N. Kinematic analysis of the cervical spine following implantation of an artificial cervical disc. Spine (Phila Pa 1976) 2005;30(17):1949-54.

16. Robertson JT, Papadopoulos SM, Traynelis VC: Assessment of adjacent-segment disease in patients treated with cervical fusion or arthroplasty: a prospective 2-year study. J Neurosurg Spine 3:417-423, 2005.

17. Mehren C, et al. Heterotopic ossification in total cervical artificial disc replacement. Spine (Phila Pa 1976) 2006;31(24):2802-6.

18. Staudt, Duggal, Cervical Disc Arthroplasty: An Overview of Past, Present, and Future. J Nervous Sys Surgery 2014; 4(4):155-168.

19. Mattei TA, \& Rehman AA: Schmorl's nodes: current pathophysiological, diagnostic, and therapeutic paradigms. Neurosurg Rev 37:39-46, 2014. 